Cahiers $d u$ MONDE RUSSE

\section{Cahiers du monde russe}

Russie - Empire russe - Union soviétique et États indépendants

$43 / 4 \mid 2002$

Intellectuels et intelligentsia

\title{
Donald J. Raleigh, ed., Provincial landscapes
}

\section{Martine Mespoulet}

\section{OpenEdition \\ Journals}

Édition électronique

URL : https://journals.openedition.org/monderusse/4048

DOI : 10.4000/monderusse.4048

ISSN : $1777-5388$

Éditeur

Éditions de l'EHESS

Édition imprimée

Date de publication : 30 décembre 2002

Pagination : 774-776

ISBN : 2-7132-1796-2

ISSN : $1252-6576$

\section{Référence électronique}

Martine Mespoulet, «Donald J. Raleigh, ed., Provincial landscapes », Cahiers du monde russe [En ligne], 43/4 | 2002, mis en ligne le 17 juin 2009, consulté le 03 septembre 2022. URL : http:// journals.openedition.org/monderusse/4048; DOI : https://doi.org/10.4000/monderusse.4048

Ce document a été généré automatiquement le 3 septembre 2022.

Tous droits réservés 


\title{
Donald J. Raleigh, ed., Provincial landscapes
}

\author{
Martine Mespoulet
}

\section{RÉFÉRENCE}

Donald J. RALEIGH, ed., Provincial landscapes. Local dimensions of Soviet power, 1917-1953. Pittsburgh, University of Pittsburgh Press, 2001, $407 \mathrm{p}$.

1 L'ouvrage Provincial landscapes : local dimensions of Soviet power, 1917-1953, publié sous la direction de Donald J. Raleigh, réunit différents textes de communications présentées au colloque organisé sur le même thème à l'université de Chapel Hill (Caroline du Nord) en avril 1999. À partir de travaux de recherche effectués dans des archives régionales, les contributions retenues éclairent différents moments de la construction du pouvoir soviétique, de la révolution d'Octobre 1917 à la mort de Stalin, en 1953.

Dès l'introduction, D. J. Raleigh expose l'enjeu méthodologique et heuristique d'un tel choix éditorial: montrer la pertinence de l'apport de l'histoire locale dans la compréhension de la mise en place et du fonctionnement du pouvoir de l'État soviétique pendant la période étudiée. À ce propos, il souligne la faible place occupée jusqu'ici par de tels travaux dans l'historiographie de l'URSS, contrairement à celle des États-Unis ou de certains pays européens, comme l'Italie ou la France. Dans le cas de l'URSS, les historiens occidentaux ont longtemps privilégié l'étude du fonctionnement du pouvoir d'État au niveau central, épousant ainsi de fait la représentation donnée par le modèle centralisé de l'État soviétique et de ses administrations. En centrant ainsi leur regard, ils ont laissé de côté l'échelle la plus proche de la population, celle du local, et n'ont pas questionné la manière dont la relation à l'État et au pouvoir pouvait se construire à la base des administrations et des entreprises d'état, dans les régions et leurs échelons territoriaux urbains et ruraux. Si la prédominance du modèle totalitaire peut expliquer une telle situation jusque dans les années $1970^{1}$, on doit noter néanmoins que l'échelon local a été peu observé dans les travaux qui ont suivi. Jusqu’à la fin des années 1980, 
l'histoire locale est restée un genre marginalisé, constitué principalement de monographies effectuées par des historiens soviétiques habitant en province sur des thématiques largement définies au niveau central, la révolution de 1917 ou l'histoire de l'organisation du parti dans une ville ou une région, par exemple.

3 L'ouverture plus large des archives de la période soviétique, à partir de 1991, aux chercheurs russes et étrangers, à Moscou et dans les régions, a stimulé de nouvelles perspectives de recherche, notamment à l'échelle locale. Ces dernières années, de nombreuses thèses ont été soutenues, en Russie et à l'étranger, sur des objets étudiés sur la base de matériaux conservés dans des archives régionales. Les contributions réunies par D. J. Raleigh ont été écrites par des historiens russes, ukrainiens et américains, jeunes ou confirmés, à partir des résultats de travaux récents. Tous ces chercheurs se sont efforcés d'analyser les phénomènes observés à l'échelle locale en interaction avec les interventions du Centre, enrichissant ainsi notre compréhension du fonctionnement du pouvoir central. L'étude minutieuse des faits et des formes concrètes des relations entre acteurs locaux et représentants du Centre les a contraints souvent à manier une approche pluridisciplinaire pour mieux appréhender la complexité de la construction des actes et des décisions de responsables ou de citoyens ordinaires. Ce faisant, leurs travaux répondent à l'intérêt grandissant pour l'étude de la vie quotidienne et des comportements des individus face au pouvoir en URSS.

Dans cette logique, l'ouvrage dirigé par D. J. Raleigh ne prétend pas proposer une histoire de la périphérie soviétique entre 1917 et 1953, mais plutôt différentes facettes de celle-ci qui renouvellent notre questionnement et, plus largement, l'interprétation des formes d'expression du pouvoir en URSS. Les textes réunis portent principalement sur la construction des identités sociales, identités de classe, de genre, mais aussi identités locales ou nationales, sur la mise en œuvre à l'échelle locale du projet politique de transformation culturelle de la société, et sur les formes des relations entre le Centre et la périphérie.

Le local est défini de manière dynamique, comme le produit des diverses relations sociales qui traversent des lieux spécifiques de multiples façons. Cette définition amène les auteurs à étudier l'organisation de la vie sociale sur un territoire administratif donné, sans négliger pour autant ses liens avec la société globale. Les territoires retenus varient de l'échelle d'une ville (Smolensk, Saratov, Iaroslavl, Rostov, Sébastopol, Samara), à celle d'une province (Tambov, Saratov), d'une république (Kazakhstan, Ukraine, Ouzbékistan), ou d'un vaste ensemble régional (Oural, Extrême-Orient, Asie centrale).

6 Les deux premières contributions portent sur la révolution de 1917 dans la province de Smolensk. Michael C. Hickey montre comment l'argument des conditions de la vie quotidienne remplaça celui de la lutte du prolétariat dans la politisation de la population locale d'une ville non industrielle et non ouvrière comme Smolensk. Pour sa part, Roberta T. Manning essaie de comprendre comment, dans un district rural dans lequel le parti bolchevik n'était pas implanté, $75 \%$ de ceux qui participèrent aux élections de l'Assemblée constituante ont néanmoins voté pour ses candidats. Cette question la conduit à mettre en évidence le rôle joué par les othodniki, paysans qui allaient travailler temporairement comme ouvriers en ville, dans la diffusion à la campagne des idées révolutionnaires, et ceci dès la révolution de 1905.

7 Dans son analyse de l'organisation des réquisitions agricoles dans la province de Tambov pendant la guerre civile, Delano DuGarm dévoile comment, loin d'obéir strictement aux ordres du Centre, les agents de l'État chargés de ces prélèvements durent composer avec les 
réactions des paysans et des acteurs politiques locaux. Les luttes entre ceux-ci et les émissaires de Moscou, les négociations entre les paysans et les agents chargés des réquisitions, les menaces et les pots-de-vin aboutirent à construire un système de prélèvements peu conforme à celui fixé par le Centre. L'étude de Donald Raleigh sur les grèves ouvrières et les révoltes paysannes dans la province voisine de Saratov, au début de l'année 1921, montre que cette crise sociale avait une signification profondément politique, d'opposition au pouvoir bolchevik, et n'était pas liée seulement aux conditions économiques du moment. Parlant d'un " Kronstadt en province ", l'auteur estime qu'elle a revêtu une dimension bien plus large que cela n'a été présenté jusqu'ici et que les historiens n'ont pas prêté suffisamment attention au rôle particulièrement important qu'elle a joué pour précipiter la mise en place des premières mesures de la NEP dans cette région.

8 Les chapitres concernant la période de la NEP analysent la manière dont l'État soviétique a cherché à remodeler la société en impulsant une politique de transformation culturelle à différentes échelles. Le cas, étudié par James T. Andrews, de la transformation d'une société scientifique locale, la Société d'histoire naturelle de Iaroslavl, est riche d'enseignements. En premier lieu, l'auteur montre clairement que le processus de centralisation par l'État de ce type de société a commencé dès le milieu des années 1920, avant donc la période à proprement parler de la révolution culturelle. Il met aussi clairement en évidence tout le jeu de négociations et de compromis développé par les responsables de cette société, à Iaroslavl, avec leur commissariat de tutelle, celui de l'Instruction, pour essayer de sauver ce qu'ils jugeaient essentiel dans leur activité. La fermeture de la société en 1931 fut la réponse ultime du pouvoir.

9 D'autres contributions s'attachent à analyser les effets de la politique menée par l'État soviétique pour transformer la société par la culture. Douglas T. Northrop étudie comment les campagnes lancées en Ouzbékistan par le parti, en 1927, contre le port du voile par les femmes eurent pour résultat de renforcer les résistances de la société locale en les cristallisant autour de l'argument d'une culture «traditionnelle» ouzbek, contribuant ainsi à construire une part de l'identité ouzbek. L'étude d'Irina Korovushkina Paert sur l'échec de la politique du parti communiste à l'égard des vieux-croyants pendant les années 1930 dans la région de l'Oural éclaire, de son côté, la manière dont la piété populaire a été un facteur essentiel de construction d'une identité locale. Offrant un autre éclairage sur cette période, David R. Shearer analyse les efforts de l'État et du parti pour introduire une "organisation socialiste» dans la Sibérie extrême-orientale. Là, c'est une guerre, usant de la violence et de la coercition, qui fut menée contre ce qui était désigné par le pouvoir comme de l'arriération culturelle.

Dans les textes relatifs à la période des années 1940 à 1953, Paula A. Michael étudie les effets de la campagne de mobilisation de l'État pendant la Seconde Guerre mondiale dans une république non russe, le Kazakhstan; Jeffrey W. Jones met en évidence les bases sociales du fonctionnement de l'économie de l'ombre dans la ville de Rostov-sur-le Don entre 1945 et 1948 ; Serhy Yerelchyk se penche sur la signification prise par la campagne de la ždanovščina en Ukraine en 1946-1948, Karl D. Quall analyse la place prise par les divers enjeux sociaux dans les différends et les négociations entre acteurs locaux et représentants du Centre au sujet du projet de reconstruction de la ville de Sébastopol pendant les années 1944-1953. Enfin, le travail d'Elena Iarskaia-Smirnova et de Pavel Romanov clôt cet ensemble avec une analyse d'entretiens menés auprès d'habitants de Saratov et de Samara au sujet de leur expérience de la vie quotidienne entre 1940 et la mort de Stalin. Il les conduit à conclure que les notions mêmes de Centre et de périphérie 
avaient des sens multiples selon la sphère d'action ou de discours, géographique, politique, sociale ou culturelle. Le Centre n'était pas perçu de la même manière selon les préoccupations et les intérêts en jeu.

11 Loin de nous éloigner du Centre, les différentes questions posées par les contributions de cet ouvrage nous y ramènent donc, soulevant en particulier celle de la place, dans la construction de cette notion, des représentations de ceux qui habitaient dans ce qui, au fil de ces divers textes, paraît être appelé à tort la périphérie.

\section{NOTES}

1. On peut mentionner toutefois quelques exceptions, notamment l'ouvrage de Merle Fainsod, Smolensk à l'heure de Staline, Paris, Fayard, 1967. L'ouvrage était paru aux États-Unis en 1958. 